\title{
A new oxygen prescription produces real improvements in therapeutic oxygen use
}

\author{
James Rudge, Sunita Odedra, Danielle Harrison \\ Sandwell and West Birmingham Hospitals NHS Trust
}

\begin{abstract}
In the UK, safe use and administration of oxygen therapy was unsatisfactory prior to the implementation of national guidelines in 2008. Each year since then the British Thoracic Society (BTS) has conducted a national audit that has demonstrated a slow but steady improvement in oxygen use across four key standards. Sandwell and West Birmingham NHS Hospitals Trust has participated in this audit process but has failed to show consistent improvements. The aim of this quality improvement project was to produce meaningful and sustained improvements in oxygen use across each of the four standards.
\end{abstract}

Four interventions were developed over three PDSA cycles and included: 1. a new oxygen prescription chart, 2. oxygen 'alert' stickers for use on drug and MEWS charts, 3. point of care resources, and 4. senior led educational sessions for healthcare staff. Each intervention was tested on the Acute Medical Unit over seven days and data collected using the BTS data collection form.

The QIP improved oxygen use across each of the standards: baseline measurement for standard one demonstrated that $55 \%$ of patients using oxygen had a valid oxygen prescription, improving to 94\% after PDSA cycle three. For standard two, baseline measurement demonstrated that $50 \%$ of patients had a documented oxygen target saturation range, improving to $94 \%$ after PDSA cycle three. For standard three, baseline measurement demonstrated that $84 \%$ patients using oxygen had saturations documented on the MEWS chart, improving to $100 \%$ after PDSA cycle three. Finally, baseline measurement of standard four demonstrated that $0 \%$ patients with a valid oxygen prescription had it signed for at drugs rounds, improving to $18 \%$ after PDSA cycle three.

Oxygen use was substantially improved during the QIP. Following engagement with stakeholders a new oxygen prescription will be rolled out within the Trust with projected annual savings of $£ 30,400$.

\section{Problem}

Therapeutic oxygen is a drug that can be lifesaving. Tissue hypoxia occurs within four minutes of inadequate ventilation, gas exchange, or circulatory distribution [1]. Supplemental therapeutic oxygen increases oxygen delivery by maximising blood oxyhaemobglobin and by raising the amount of oxygen dissolved in plasma [1]. This is important for a wide variety and large number of acutely unwell patients with hypoxaemia caused by pneumonia and atelectatic lung, alveolar hypoventilation such as in opioid overdose, right to left shunting due to pulmonary embolism, and carbon monoxide poisoning.

Conversely, excessive amounts of oxygen can be dangerous in patients at risk of hypercapnic respiratory failure due to chronic pulmonary, neuromuscular and thoracic wall disease $[1,2]$. In the acutely unwell patient in the non-emergency setting arterial blood gases (ABG) should be performed to assess the risk of hypercapnic respiratory failure and oxygen titrated accordingly. This is particularly true for patients with COPD, since a proportion of these will not be at risk of hypercapnia. Other dangers of oxygen therapy include combustion, damage to the alveolar membrane, and in hyperbaric concentrations cerebral vasoconstriction and epileptic fits.
Every year incidents relating to oxygen use are reported to the National Patient Safety Agency (NPSA) [3]. Between 2004 to 2009, 281 serious incidents were identified as a result of inappropriate oxygen therapy. Of these, 75 were associated with prescribing issues; nine may have directly caused a patient's death and 35 may have contributed to death. The NPSA concluded these deaths may have been prevented with better oxygen prescribing practice.

Accurate oxygen prescription is an important step in minimising harm and ensuring safe and effective use in the acutely ill patient. However, it is recognised that doctors prescribe oxygen poorly [1]. In New Zealand, oxygen prescribing practice amongst hospitalised patients has been shown to be poor [4]. Subsequent interventions including the introduction of local guidelines have made little impact [5]. In a Canadian study, prescribing practice for therapeutic oxygen was worse than for antibiotics and attributed to a lack of appreciation of oxygen as a medical drug [6].

A similar pattern of behaviour towards oxygen prescribing practice has been shown in the United Kingdom [7]. However, certain interventions including the introduction of specific oxygen prescription cards at a specialist respiratory centre in Manchester have produced significant improvements in the incidence, accuracy, and validity of oxygen prescription amongst inpatients [7]. Whilst there is good evidence to suggest that educational activities in 
BMJ Quality Improvement Reports

isolation do not improve prescribing practice, most studies acknowledge the importance of this in supporting other interventions [2-7].

In the UK, safe use of oxygen therapy was highly unsatisfactory prior to the implementation in 2008 of national guidelines [1]. Since then, audit data from the British Thoracic Society (BTS) has demonstrated a slow but steady improvement in oxygen use [8]. Baseline data on oxygen use in Sandwell and West Midlands NHS Hospitals Trust, which comprises two large teaching hospitals, was measured in 2010. This demonstrated poor results compared to other hospitals nationally.

To help improve oxygen use within the Trust the quality improvement project (QIP) team asked the following: what are the reasons for poor oxygen use within the Trust? New interventions were developed around this question.

\section{Background}

Oxygen is a treatment for hypoxaemia and its safe and effective use is set out in national guidelines devised by the BTS and implemented in 2008 [2, 8]. The guideline can be summarised as "a requirement for oxygen to be prescribed according to a target saturation range and for those who administer oxygen therapy to monitor the patient and keep within the target saturation range."

A key objective of the BTS was to audit oxygen use in the UK before implementation of the guidelines, and annually thereafter. Four standards, each with a target of $100 \%$, were identified:

1. Unless in emergency for the critically ill patient, when its use should be documented after administration, oxygen should be prescribed on a designated drug chart or oxygen order

2. The prescription should include a documented target saturation range of $94-98 \%$ for most acutely ill patients or $88-92 \%$ for those at risk of hypercapnic respiratory failure

3. Oxygen saturation should be documented on the patient's observations chart at each observations round

4. Oxygen should be signed for on the drug chart on each drug round

The results of the BTS audits in 2008 (prior to implementation of the guideline) and 2010 (after implementation) demonstrated that the number of patients using oxygen fell from $17.5 \%$ to $15.5 \%$, whilst the number with a prescription rose from $32 \%$ to $56 \%$ [8]. Of those with a prescription, only $10 \%$ had a documented target range in 2008 , rising to $41 \%$ by 2010 . Documentation of oximetry on observation charts was $94 \%$ in 2008 and improved to $99 \%$ in 2010 . Oxygen was rarely signed for at drug rounds, rising from $5 \%$ in 2008 to $16 \%$ in 2010 .

Despite the above modest improvements, the authors of these studies noted that difficulty in changing established practice was the main reason that more rapid improvements in oxygen use were not observed [8]. Several explanations for this were suggested including poor quality oxygen prescription and bedside observation charts, and inadequate training of healthcare professionals [8].

\section{Baseline measurement}

The initial Trust baseline measurement was undertaken in 2010 by junior doctors working within the respiratory department under the supervision of two senior consultants. Data was collected using the BTS emergency oxygen audit data collection form during an eightweek period and returned to the BTS for analysis; all eligible patients were included to minimise selection bias. Results were returned at local (hospital) and national level.

In 2010 , oxygen use in the Trust was $13 \%$ compared to $15 \%$ nationally. Baseline data for each of the four standards is presented below (national data):

1. Valid oxygen prescription: $55 \%(56 \%)$ ?

2. Documented target saturation range: $50 \%(44 \%)$ ?

3. Oxygen saturations documented at observations rounds: $84 \%(99 \%)$

4. Oxygen signed for at each drugs round: $0 \%(16 \%)$

\section{Design}

The QIP team felt that a major problem contributing to poor oxygen use within the Trust was a lack of understanding about good prescribing practice by healthcare professionals. In particular, stakeholders highlighted the need to target those individuals responsible for the majority of oxygen prescriptions, namely junior doctors. To address this, a new educational programme was devised and delivered over two weeks at the beginning of August 2012 (PDSA cycle 1) to coincide with induction of new doctors. This consisted of didactic sessions utilising powerpoint presentation. A Trust-wide email campaign was also used to disseminate audit findings and raise awareness amongst other healthcare professionals. Interventions would be continued annually with each influx of new doctors. Although time consuming, this would ensure other healthcare professionals who remain within the Trust continue to receive regular education about oxygen use. The following interventions were devised:

1. Formal lecture series to all new doctors at induction: designed to explain the current oxygen prescription chart, how to complete a valid oxygen prescription in line with the above standards, how to select appropriate oxygen delivery devices and initial flow rates, and how to identify patients at risk of hypercapnic respiratory failure

2. Production and Trust-wide dissemination of educational materials to doctors, nurses and auxiliary staff including baseline audit data, simple steps to ensure safe use of oxygen, and information about further e-Learning resources

Results of the 2013 BTS audit demonstrated a worsening of oxygen use within the Trust. It was agreed that to produce meaningful improvements a change would need to be made to the oxygen prescription chart. To support this and facilitate a change in culture towards oxygen use new educational materials would be produced. 
The following interventions were developed:

1. A new oxygen prescription: designed to i) simplify the process of writing a prescription, and ii) improve compliance with guideline standards. Essential information, such as the desired target saturation range, was included as tick box options whilst unnecessary content was removed and the layout adjusted to improve continuity with regular prescription boxes. The new oxygen prescription was designed as a sticker and placed over the existing one

2. New oxygen 'alert' stickers: small $5 \times 3 \mathrm{~cm}$ stickers for use on the front of drug and MEWS charts to overcome issues raised by nursing staff about the difficulty in crossreferencing the prescribed oxygen target saturation range on the drug chart with the achieved oxygen saturation that is documented on the MEWS chart. The expectation was that healthcare professionals involved in these activities would apply the stickers themselves

3. New point of care educational material: large colour posters outlining a simple four step process to ensure effective use of oxygen in line with the above standards. Posters were positioned in areas of high clinical traffic with links to further sources of information. Versions of the poster were used during educational sessions for healthcare professionals and disseminated via email

4. Education by senior doctors and nursing staff: to occur in the clinical environment at key times during the day, for example during hand over. Education was intended to highlight the use of the new interventions and to answer any questions. Use of senior staff was intentional and expected to improve credibility of the QIP

Interventions were circulated for feedback to stakeholders comprising acute medicine and respiratory physicians as well as senior nursing staff. After suggestions were incorporated it was agreed that interventions would be tested over seven days on the acute medicine unit (AMU).

An educational campaign was commenced two weeks before the prospective audit and continued throughout the period of data collection. This involved teaching sessions to medical and nursing staff about the new interventions. Meetings were also held with senior nursing staff and consultants to reinforce their role in supporting the QIP.

\section{Strategy}

PDSA cycle 1: educational sessions were undertaken in August 2012 as part of the Trust induction of new doctors. An email campaign was disseminated to all healthcare professionals and included a summary of the 2010 audit results as well as a poster explaining how to use oxygen effectively. Subsequent audit failed to demonstrate any improvement across each of the four measured standards. The main problem was felt to be that the old oxygen prescription was not fit for purpose and that revisions in this would lead to more sustained changes, particularly with respect of standards one and two, which are largely the responsibility of doctors. Additional interventions would be required to address standards three and four, which are largely the responsibility of the nursing team. All new interventions were signed off by senior stakeholders and education delivered by them either in person or electronically.

PDSA cycle 2: the new materials were distributed to consultants and senior nursing staff and qualitative data was used to make several revisions. The revised materials were placed on the AMU and used by the clinical team over three days. Uptake of the new oxygen prescription chart was good however, old versions were still used as too few new charts had been provided. From this data the minimum number of new oxygen prescription charts required for the seven-day audit period was calculated. Uptake of the oxygen alert sticker was very poor; junior medical and nursing staff reported that they did not know how to use it or were unaware that it existed. Staff indicated that the point of care materials were useful but that many had been removed or obscured from view.

PDSA cycle 3: an education campaign led by senior nursing and medical staff was commenced two weeks prior to the implementation of new oxygen prescription charts, oxygen alert stickers, and point of care literature. Working groups during weekly departmental teaching sessions were held with junior medical staff and questions relating to the audit answered. Concurrently, new materials were discussed during each nursing handover with emphasis on the use of oxygen alert stickers and the importance of signing for oxygen at drugs rounds. New oxygen prescription charts were placed in the usual locations on the AMU and used continuously for seven days; prospective data was collected daily at 18:00. All point of care literature was repositioned and porters and auxiliary healthcare staff requested not to remove or obscure them. An accompanying email was sent to the junior doctor team to ensure those working on call during the audit were aware of the new materials.

See supplementary file: ds4004.docx - "PDSA Cycle Summary"

\section{Post-measurement}

A summary of the results are presented in figure 1. Baseline measurement of standard one demonstrated that $55 \%$ of patients using oxygen had a valid oxygen prescription, falling to $54 \%$ after PDSA one but improving to $94 \%$ after PDSA cycle three.

For standard two, baseline measurement demonstrated that $50 \%$ of patients had a documented target oxygen saturation range, falling to $39 \%$ after PDSA cycle one but improving to $94 \%$ after PDSA cycle three.

For standard three, baseline measurement demonstrated that $84 \%$ of patients using oxygen had saturations documented on the MEWS chart, improving to $94 \%$ and $100 \%$ after PDSA cycles one and three, respectively.

Finally, baseline measurement of standard four demonstrated that $0 \%$ of patients with a valid oxygen prescription had it signed for at drugs rounds. This improved to $5 \%$ and $18 \%$ after PDSA cycles one and three, respectively. 
BMJ Quality Improvement Reports

See supplementary file: ds4309.docx - "Figure 1. Compliance of Trust oxygen prescribing practice with BTS guidelines. "

\section{Lessons and limitations}

We encountered several challenges during the QIP. Firstly, the project was undertaken over 24-months and several members of the team left during this time. New members were less experienced with the project and were unfamiliar with Trust guidelines. To minimise the impact of this problem the project lead was selected on the basis of their involvement from the beginning. They were responsible for ensuring understanding, task delegation and timely completion of the project. They also acted as a single point of contact between team members and stakeholders. It is important to note that given the long duration of this study, the improvements noted may have occurred due to incidental improved awareness by junior doctors about effective oxygen prescribing practice. However, the poor results demonstrated after PDSA 1 in combination with the short interval to completion of PDSA 3 less than six months later (i.e. within the same cohort of doctors) suggests that it was the QIP interventions that contributed to the improvements observed.

The QIP did not look at the impact of the new interventions beyond the AMU. A fourth cycle of PDSA on another ward or area of the hospital would have strengthened the results. Also, as the new oxygen prescription chart will not be printed and used until early 2015 we have not been able to assess its impact. Whilst it is anticipated that improvements will be significant, data will not be available until 2015.

A key challenge faced by the project team was collecting data for the BTS audit across the Trust over two large teaching hospitals. Consequently two sub-teams each with responsibility for one site were used with a single point of contact through the project leader. However, due to logistical constraints the 2014 QIP was undertaken on one site only; data therefore only reflects this site. This is a challenge affecting many Trusts and novel approaches to communication, including digital meetings, will help to negate these difficulties.

The 2014 QIP utilised four interventions to improve oxygen use within the Trust. Of those interventions, the authors found that the oxygen alert sticker was not used effectively despite additional education being provided after findings from PDSA cycle two. It was hoped that the sticker would be applied to both the drug and MEWS charts to inform healthcare professionals, primarily nurses, that the patient was using oxygen and it should be titrated and signed for. Qualitative data suggested that healthcare professionals were unclear about who was expected to attach the sticker to charts as well as what it was intended do. Further education will be required about this.

Finally, despite additional education about this problem after PDSA cycle two, point of care educational materials continued to be removed or obscured from view. Qualitative data suggested that when observed, point of care material was useful, clear, and informative. When completing a project involving this type of intervention, it is important to identify appropriate areas that will be accessible at all times.

\section{Conclusion}

Medical Oxygen is a drug. The BTS requires that oxygen be prescribed according to a target saturation range and for those who administer oxygen therapy to monitor the patient and keep within the target saturation range.

The 2014 QIP was devised following the recognition that oxygen use within the Trust did not meet required national standards. Over several PDSA cycles, carried out between 2012 and 2014, we implemented several new interventions that resulted in substantial improvements in the use of medical oxygen therapy. The negative impact of poor oxygen therapy is likely to have reduced as a result of these improvements however it is not possible to quantify this effect directly. Although not the main aim of this QIP, it is estimated that these results equate to an annual financial saving of $£ 30,400$.

The 2014 QIP demonstrates the importance of creating interventions that produce sustained results. It also highlights the importance of targeting all healthcare professionals involved in medical oxygen therapy and not individual groups such as doctors. A further cycle of audit in 2015 will provide data about the impact of the new oxygen prescription chart. The results of this audit will identify where further improvements can be made.

\section{References}

1. Bateman NT, Leach RM. ABC of Oxygen. Acute oxygen therapy. BMJ. 1998;317:798-801

2. O'Driscoll BR, Howard LS, Davison AG. BTS guideline for emergency oxygen use in adult patients. Thorax 2008;63(Suppl VI):vi1-vi68

3. National Patient Safety Agency. Oxygen safety in hospitals: Rapid Response Report. 2009. http://www.nrls.npsa.nhs.uk/resources/?entryid45=62811. Accessed 15th October 2014

4. Boyle M, Wong J. Prescribing oxygen therapy. An audit of oxygen prescribing practices on medical wards at North Shore Hospital, Auckland, New Zeal Z Med J. 2006;119:U2080

5. Kbar FA, Campbell IA. Oxygen therapy in hospitalized patients: the impact of local guidelines. J Eval Clin Pract 2006;12:31e6

6. Small D, Duha A, Wieskopf B, et al. Uses and misuses of oxygen in hospitalized patients. The American Journal of Medicine. 1992; 92;6:591-595

7. Dodd ME, Kellet F, Davis A, et al. Audit of oxygen prescribing before and after the introduction of a prescription chart. BMJ. 2000; 321:864-865

8. O'Driscoll BR, Howard LS, Bucknall C, Welham SA, Davison AG; British Thoracic Society. British Thoracic Society emergency oxygen audits. Thorax. Aug;66(8):734-5

\section{Declaration of interests}


Nothing to declare

\section{Acknowledgements}

The team would like to thank Dr O. Khair, Consultant Respiratory Physician, Sandwell and West Birmingham Hospitals NHS Trust, for his supervision and guidance. 